\title{
Geant4 Maintainability Assessed with Respect to Software Engineering References*
}

\author{
Elisabetta Ronchieri ${ }^{\dagger} \quad$ Maria Grazia Pia ${ }^{\ddagger} \quad$ Tullio Basaglia ${ }^{\S} \quad$ Marco Canaparo $₫$
}

April 21, 2017

\begin{abstract}
We report a methodology developed to quantitatively assess the maintainability of Geant 4 with respect to software engineering references. The level of maintainability is determined by combining a set of metrics values whose references are documented in literature.
\end{abstract}

${ }^{*}$ Presented at 2016 IEEE NSS/MIC - Strasbourg, France, 29 October - 5 November, 2016

${ }^{\dagger}$ INFN CNAF at Bologna. Email: elisabetta.ronchieri@cnaf.infn.it

${ }^{\ddagger}$ INFN at Genoa. Email: maria.grazia.pia@ge.infn.it

${ }^{\S}$ CERN. Email: Tullio.Basaglia@cern.ch

"INFN CNAF at Bologna. Email: marco. canaparo@cnaf.infn.it 


\section{Introduction}

This report documents what has been done so far to assess Geant4 maintainability - one of software characteristics defined in software quality standards, such as ISO/IEC 25010:2011 (former ISO/IEC 9126) [ISO11.

Maintainable software allows development teams to fix bugs, add new features, improve usability and increase performance. Organizations that deal with software in different domains, such as telecommunications, aerospace and simulations, monitor such software characteristic to maintain skills and knowledge in order to understand and make changes to their software.

Software characteristics are measured by metrics values. We identified and assessed software metrics tools (both free and under commercial licenses) to collect a large number of measurements [RPG14], RPG15]. As a result of this assessment, we selected Imagix4D [Ima]: this tool measures several product metrics at different levels, such as file, class, directory, namespace, function and variable, and its vendor positively collaborates with research communities. Metrics used in this study provide code information about size, coupling, inheritance, control-flow structuredness, cohesion, staticness. Existing literature gives references of such metrics for different programming languages. We identified $\mathrm{C}++$ metric thresholds to determine the goodness of code.

\section{Method}

The methods used to perform this maintainability assessment is characterized by the following steps:

1. collecting the source code of all Geant $4\left[\mathrm{AAA}^{+} 03\right.$, AAea06] versions from 0 to the current one $(10.2)$;

2. loading the Geant4 source code into Imagix $4 \mathrm{D}$ version 8.0.4 to measure a large number of metrics in order to obtain code information about size, coupling, inheritance, control-flow structuredness, cohesion, staticness;

3. saving all the collected data at different levels of granularity, such as file, function, class, directory, variable and namespace;

4. application of statistical methods for the analysis of metric values;

5. identification of quality (goodness ranges of) references with respect to size, coupling, inheritance, control-flow structuredness, cohesion, staticness, derived from relevant peer-reviewed papers, conference proceedings and technical reports [ $\mathrm{RC} 16$.

Some of the metrics we collected are listed in Tables 1, 2 and 3 ,

Size metrics [LK94 quantify code size. They are estimators of software cost and effort.

Complexity metrics, such as McCabe [McC76] and Halstead [Hal77] metrics, measure the simplicity of the system design. McCabes complexity, also called cyclomatic complexity, quantifies the control flow within a program by counting the independent paths on a control flow graph. The path indicates a certain degree of well structuredness of an application.

Object-oriented metrics CK94 measure complexity, maintenance and clarity; they estimate to which extent the system adheres to the object orientation. 
Table 1: Some Metrics of the Size Group

\begin{tabular}{lll}
\hline Group & Size Metric & Source \\
\hline \multirow{7}{*}{ File } & Comment Ratio & Lorenz and Kidd \\
& Declarations in File & Lorenz and Kidd \\
& File Size & Lorentz and Kidd \\
& Functions in File & Lorenz and Kidd \\
& Lines in File & Lorenz and Kidd \\
& Lines of Source Code & Lorenz and Kidd \\
& Lines of Comments & Lorenz and Kidd \\
& Number of Statements & Lorenz and Kidd \\
& Variables in File & Lorenz and Kidd \\
\hline \multirow{3}{*}{ Function } & Lines in Function & Lorenz and Kidd \\
& Lines of Source Code & Lorenz and Kidd \\
& Variables in Function & Lorenz and Kidd \\
\hline & &
\end{tabular}

Table 3: Some Metrics of the Object-Oriented Group

\begin{tabular}{lll}
\hline Group & Object-Oriented Metric & Source \\
\hline \multirow{4}{*}{ Class } & Class Cohesion (LCOM) & Chidamber and Kemerer \\
& Class Coupling (CBO) & Chidamber and Kemerer \\
& Depth of Inheritance (DIT) & Chidamber and Kemerer \\
& Number of Children (NOC) & Chidamber and Kemerer \\
& Response for Class (RFC) & Chidamber and Kemerer \\
& Weighted Methods (WMC) & Chidamber and Kemerer \\
\hline
\end{tabular}

Table 2: Some Metrics of the Complexity Group

\begin{tabular}{lll}
\hline Group & Complexity Metric & Source \\
\hline File, & Intelligent Content & Halstead \\
Function, & Mental Effort & Halstead \\
Class & Program Volume & Halstead \\
& Program Difficulty & Halstead \\
\hline File, & Average Cyclomatic Complexity & McCabe \\
Class & Maximum Cyclomatic Complexity & McCabe \\
& Total Cyclomatic Complexity & McCabe \\
\hline File & Maintainability Index & Welker \\
\hline \multirow{5}{*}{ Function } & McCabe Cyclomatic Complexity & McCabe \\
& McCabe Decision Density & McCabe \\
& McCabe Essential Complexity & McCabe \\
& McCabe Essential Density & McCabe \\
\hline
\end{tabular}

\section{A sample of quality references and results}

Figure 1 shows the trend of McCabe Maximum Cyclomatic Complexity at class level for Geant4 hadronic physics diffraction package, while Figure 2 shows the trend of Halstead' programme volume for the electromagnetic physics xrays package.

Table 4 shows a sample of quality references.

\section{Conclusions}

The use of metrics can contribute to monitor the internal quality of software. Further investigation is in progress to identify appropriate ranges of metric values for Geant4 packages by using statistical methods. More extensive results will be discussed in a forthcoming full paper.

\section{Acknowledgment}

The authors thank Francesco Giacomini for interesting discussions and INFN CCR for partly funding this work. We also thank the Imagix Corporation that provided an extended free full license of Imagix $4 \mathrm{D}$ for performing this work and CERN library for providing papers and books. 


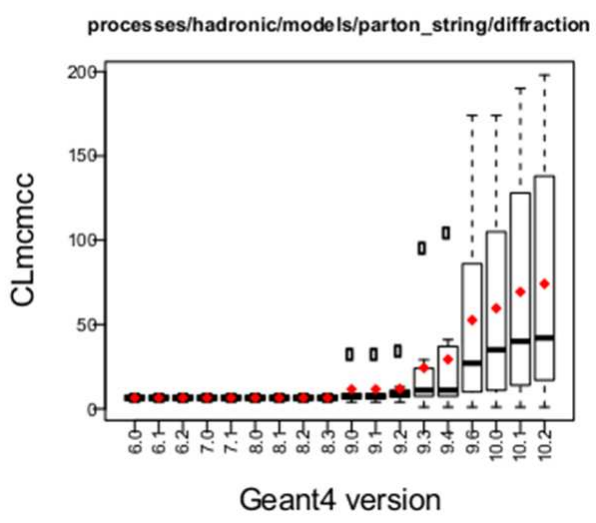

Figure 1: McCabe Maximum Cyclomatic Complexity at class level for the diffraction package.

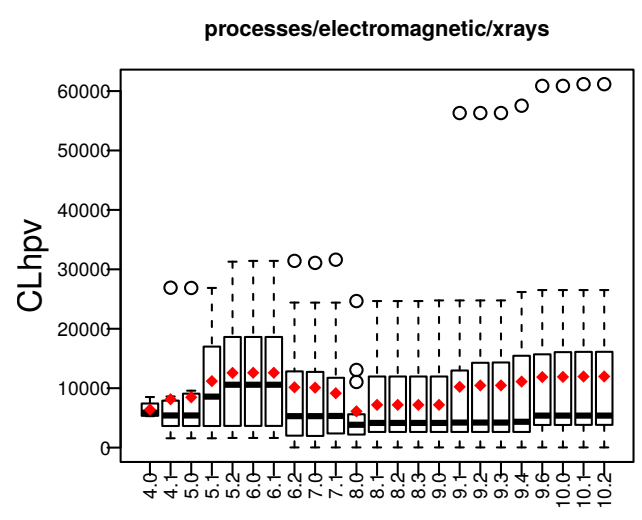

Geant4 version

Figure 2: Halsteads programme volume at class level for the xrays package.

Table 4: A Sample of Quality References

\begin{tabular}{|c|c|c|}
\hline Acronym & Reference & Source \\
\hline Comment Ratio & 0.08 & McCabe \\
\hline SLOC (Source Lines Of Code) & 60 at file level & McCabe \\
\hline \multirow{5}{*}{ HPV (Halstead Programme Volume) } & 1500 at function level & McCabe \\
\hline & {$[100,8000]$ at file level } & Verysoft Technology Tec \\
\hline & $>800$ too many things at file level & Verysoft Technology Tec \\
\hline & {$[20,1000]$ at function level } & Verysoft Technology Tec \\
\hline & $>1000$ too many things at function level & Verysoft Technology Tec \\
\hline \multirow{3}{*}{ MI (Maintainability Index) } & $<65$ poor maintainability & Coleman, Lowther, Oman CLO95 \\
\hline & {$[65,84]$ fair maintainability } & Coleman, Lowther, Oman CLO95 \\
\hline & $\geq 85$ excellent maintainability & Coleman, Lowther, Oman CLO95 \\
\hline \multirow{8}{*}{ MCMCC (McCabes Maximum Cyclomatic Complexity } & {$[1,10]$ low $\mathrm{CC}$} & CppDepend Cpp \\
\hline & {$[11,15]$ medium $\mathrm{CC}$} & CppDepend Cpp \\
\hline & {$[16,30]$ high $\mathrm{CC}$} & CppDepend Cpp \\
\hline & $>31$ very high $\mathrm{CC}$ & CppDepend Cpp \\
\hline & {$[1,10]$ low $\mathrm{CC}$} & McCabe \\
\hline & {$[11,20]$ medium $\mathrm{CC}$} & McCabe \\
\hline & [21. 50] high CC & McCabe \\
\hline & $>51$ & McCabe \\
\hline
\end{tabular}

\section{References}

$\left[\mathrm{AAA}^{+} 03\right]$ S. Agostinelli, J. Allison, K. Amako, J. Apostolakis, H. Araujo, and et al. Geant4 - a simulation toolkit. Nuclear Instruments and Methods in Physics, 506(3):250-303, July 2003.

[AAea06] J Allison, K Amako, and et al. Geant4 Developments and Applications. IEEE Transactions on Nuclear Science, 53(1):270-278, February 2006.

[CK94] S R Chidamber and C F Kemerer. A Metrics Suite for Object-Oriented Design. IEEE Transactions on Software Engineering, 20:476-493, 61994.

[CLO95] D Coleman, B Lowther, and P Oman. The application of software maintainability models on industrial software systems'. Journal System Software, 29(1):3-16, 1995.

[Cpp] CppDepend. http://www.cppdepend.com/. 
[Hal77] M H Halstead. Elements of Software Science. Elsevier, North-Holland Inc., 1977.

[Ima] Imagix. https://www.imagix.com/products/static-analysis-and-metrics.html.

[ISO11] ISO/IEC. 25010:2011 - Systems and software engineering - Systems and software Quality Requirements and Evaluation (SQuaRE) - System and software quality models, 2011.

[LK94] M Lorenz and J Kidd. Object-Oriented Software Metrics: A Practical Guide. Prentice-Hall, 1994.

[McC76] T J McCabe. A Complexity Measure. IEEE Transaction on Software Engineering, SE2(4):308-320, 1976.

[RC16] E Ronchieri and M Canaparo. A Preliminary Mapping Study of Software Metrics Thresholds. In ICSOFT-EA 2016, pages 232-240, 2016.

[RPG14] E Ronchieri, M G Pia, and F Giacomini. Software quality metrics for geant4: An initial assessment. In ANS RPSD, 2014.

[RPG15] E Ronchieri, M G Pia, and F Giacomini. First statistical analysis of geant4 quality software metrics. Journal of Physics: Conference Series, 664, 2015.

[Tec] Verifysoft Technology. http://www.verifysoft.com/en_halstead_metrics.html. 